University of Nebraska - Lincoln

DigitalCommons@University of Nebraska - Lincoln

Jay F. Storz Publications

Papers in the Biological Sciences

May 2003

\title{
Natural Selection on Protein Polymorphism in the Rodent Genus Peromyscus: Evidence from Interlocus Contrasts
}

Jay F. Storz

University of Nebraska - Lincoln, jstorz2@unl.edu

Michael W. Nachman

University of Arizona, Biosciences West, Tucson, Arizona

Follow this and additional works at: https://digitalcommons.unl.edu/bioscistorz

Part of the Genetics and Genomics Commons

Storz, Jay F. and Nachman, Michael W., "Natural Selection on Protein Polymorphism in the Rodent Genus Peromyscus: Evidence from Interlocus Contrasts" (2003). Jay F. Storz Publications. 13.

https://digitalcommons.unl.edu/bioscistorz/13

This Article is brought to you for free and open access by the Papers in the Biological Sciences at DigitalCommons@University of Nebraska - Lincoln. It has been accepted for inclusion in Jay F. Storz Publications by an authorized administrator of DigitalCommons@University of Nebraska - Lincoln. 


\title{
Natural Selection on Protein Polymorphism in the Rodent Genus Peromyscus: Evidence from Interlocus Contrasts
}

\author{
Jay F. Storz and Michael W. Nachman \\ Department of Ecology and Evolutionary Biology, University of Arizona, Biosciences West, Tucson, Arizona 85721
}

\begin{abstract}
The effects of natural selection are generally locus-specific, whereas migration, drift, and inbreeding are expected to have relatively uniform effects across the entire genome. This suggests that multilocus surveys of multiple populations can be used to distinguish selection from demographic effects. The purpose of this study was to test for evidence of selection on protein polymorphism in natural populations of mice in the genus Peromyscus. We analyzed published data from geographic surveys of allozyme variation and used a coalescent-based simulation model to identify specific loci that deviated from neutral expectations. Observed $F_{\mathrm{ST}}$ values generally exhibited a remarkably close fit to the expected neutral distributions, indicating that the majority of loci are simply tracking stochastic demographic processes. A smaller number of loci exhibited highly significant departures from the expectations of the neutral model and thus appear to be tracking the direct or indirect effects of selection. Most departures from neutrality were characterized by $F_{\mathrm{ST}}$ values that far exceeded neutral expectations and were therefore attributable to spatially varying selection. Interestingly, the albumin locus was implicated as a candidate gene for local adaptation in four different species of Peromyscus. The results also demonstrate that selection can severely bias marker-based estimates of neutral parameters.
\end{abstract}

Keywords: adaptation, albumin, allozymes, coalescent, Lewontin-Krakauer test, mice, neutral theory

At what fraction of protein-coding genes has natural selection shaped patterns of polymorphism and divergence? This question is of central importance in evolutionary genetics (Lewontin 1974; Gillespie 1991). The original neutralist-selectionist debate revolved around competing explanations for observed patterns of protein sequence divergence and allelic polymorphism. The polymorphism argument centered on explanations for the unexpectedly high levels of allelic variation revealed by electrophoretic surveys of metabolic enzymes and other soluble proteins (e.g., Harris 1966; Hubby and Lewontin 1966; Lewontin and Hubby 1966). The traditional selectionist position held that protein polymorphisms are typically maintained by some form of balancing selection that arises from overdominance of fitness (single-locus heterosis), or from spatial, temporal, or frequency-dependent variation in the relative fitness of alternative genotypes. By contrast, the neutralist position is that observed levels of protein polymorphism in natural populations are much too high to be accounted for by balancing selection, but can be easily explained by stochastic fluctuations in the frequencies of functionally equivalent alleles (Kimura and Ohta 1971). In principle, these competing hypotheses could be tested by obtaining experimental measures of fitness differences among alternative genotypes. However, even if selection coefficients for allelic protein variants are large enough to dominate genetic drift over evolutionary time scales, they may still typically lie well below the resolving power of experimental protocols (Lewontin 1974; Eanes 1987; Gillespie 1991).

An alternative approach is to use multilocus surveys of multiple populations to infer selection from patterns of geographic variation in allele frequencies. The premise of this approach is that the effects of selection are generally locus-specific, whereas migration, drift, and inbreeding are expected to have relatively uniform effects across the entire genome (Cavalli-Sforza 1966). If allelic variation at most loci is simply tracking stochastic demographic processes, loci under selection should produce a detectable signal against the genome-wide backdrop of neutral variation. For example, if multiple subpopulations are surveyed across the geographic range of a species, a locus subject to spatially varying selection is expected to exhibit greater heterogeneity in allele frequencies relative to unlinked neutral markers. Conversely, a locus subject to spatially uniform selection (i.e., due to simple overdominance of fitness) is expected to exhibit relatively less heterogeneity.

This general approach was first formalized as a statistical test by Lewontin and Krakauer (1973). In this test, the neutral expectation for the interlocus variance of $F_{\mathrm{ST}}$ is given by $2 F_{\mathrm{ST}}^{2} /(n-1)$, where $n$ is the number of subpopulations, and the observed variance is then compared with this expected value using a standard variance ratio test. This test was criticized on the grounds that the expectation for the interlocus variance in $F_{\mathrm{ST}}$ did not account for patterns of migration or population history that could induce a correlation in allele frequencies among subpopulations. For example, a spatial autocorrelation in allele frequencies could result from a stepping-stone pattern of migration (Nei and Maruyama 1975) and a phylogenetic autocorrelation in allele frequencies could result from variation in divergence times among hierarchically related subpopulations (Robertson 1975a,b). A possible solution to this problem is to use a coalescent simulation model to generate null distributions of $F_{\mathrm{ST}}$ conditional on allele frequencies for biallelic loci (Bowcock et al. 1991) or conditional on singlelocus heterozygosity for multiallelic loci like allozymes (Beaumont and Nichols 1996; see also McDonald 1994; Vitalis et al. 2001). Outlying loci can then be identified by assessing the probability of observed $F_{\mathrm{ST}}$ values under a neutral model of population structure. Of course, locus-specific 
Table 1. Datasets for Peromyscus species that were used to test for evidence of natural selection on protein polymorphism. Data are based on multilocus allozyme surveys of natural populations.

\begin{tabular}{llccc}
\hline Species & Reference & $\begin{array}{l}\text { No. sampled } \\
\text { subpopulations }\end{array}$ & $\begin{array}{l}\text { Median no. } \\
\text { mice/subpopulation }\end{array}$ & $\begin{array}{c}\text { No. polymorphic } \\
\text { loci }\end{array}$ \\
\hline P. californicus & Smith 1979 & 13 & 12 & 17 \\
P. furous & Harris and Rogers 1999 & 7 & 11 & 24 \\
P. gossypinus & Boone 1995; Boone et al. 1999 & $50^{1}$ & 25 & 37 \\
P. maniculatus & Marquardt 1976 & 7 & 30 & 15 \\
P. leucopus & Robbins et al. 1985 & $12^{2,3}$ & 22 & 10 \\
P. leucopus & Boone 1995 & 12 & 14 & 33 \\
P. polionotus & Selander et al. 1971 & $28^{4}$ & 15 & 15 \\
\hline
\end{tabular}

${ }^{1}$ Samples from islands (localities 11, 23, 28, 30, 31, 53, 55, and 63) were excluded (see appendix 2.3 in Boone 1995).

${ }^{2}$ Analysis of allozyme variation was restricted to the "Northeastern" chromosomal race of P. leucopus (see Baker et al. 1983; Robbins et al. 1985).

${ }^{3}$ Allele counts for localities 9 and 10 were pooled (see Figure 1 in Robbins et al. 1985).

${ }^{4}$ Samples from islands (localities 29 and 30) were excluded (see table 1 in Selander et al. 1971).

$P$-values only apply to the particular model used to generate the expected distribution of $F_{\mathrm{ST}}$.

The purpose of this study was to test for evidence of natural selection on protein polymorphism in natural populations of mice in the genus Peromyscus. We analyzed published data from multilocus allozyme surveys and used a coalescent-based simulation model to identify specific loci that deviate from neutral expectations. Species of Peromyscus are found throughout North America and are often distributed across ecologically heterogeneous landscapes. These mice exhibit many apparently adaptive patterns of geographic variation in morphology, physiology, and behavior (reviewed by Blair 1950). It remains to be seen whether these patterns of geographic variation are mirrored by adaptive variation in biochemical phenotypes (as revealed by allozymes).

\section{Methods}

We analyzed seven published datasets based on allozyme surveys of mice in the genus Peromyscus (Table 1). For the purposes of this analysis we only considered datasets in which 10 or more polymorphic loci were surveyed in seven or more subpopulations (with a median sample size of 10 or more mice/subpopulation). Peromyscus is an ideal taxon for this type of analysis because a number of rangewide surveys of allozyme variation have been conducted, and for many loci, codominant segregation of electrophoretic variants has been confirmed, allelic heterogeneity within electrophoretic mobility classes has been characterized, and linkage relationships have been established (e.g., Baccus et al. 1980; Aquadro and Avise 1982; Dawson 1982; Dawson et al. 1983).

\section{The Model}

After compiling allele counts for each of the seven datasets, we obtained single-locus estimates of $F_{\mathrm{ST}}$ (a standardized measure of genetic differentiation) using the $\beta$ statistic of Cockerham and Weir (1993):

$$
\beta=\left(F_{0}-F_{1}\right) /\left(1-F_{1}\right)
$$

where $1-F_{1}$ is the average probability of identity by descent for two alleles sampled randomly from separate subpopulations, and $1-F_{0}$ is the average probability of iden- tity by descent for two alleles sampled randomly from the same subpopulation. We estimated heterozygosity, $H$, as $1-F_{1}$. For each dataset, we calculated the expected $F_{\mathrm{ST}}$ as the among-locus average weighted by $H$ (Weir and Cockerham 1984). We then tested for evidence of selection by comparing observed $F_{\mathrm{ST}}$ values to a null distribution generated by a coalescent-based simulation model. Specifically, we used the model of Beaumont and Nichols (1996) to generate a neutral distribution of $F_{\mathrm{ST}}$ as a function of $H$. Coalescent simulations were performed using a symmetrical hundred-island model of population structure and the infinite alleles model of mutation. Sample sizes were set equal to the median of actual sample sizes in the specific dataset under consideration. To generate a roughly uniform distribution of $H$ values, simulations were based on a range of values for $N_{\mathrm{e}} \mu$ (the mutation rate scaled to effective population size). The scaled migration rate, $N_{\mathrm{e}} m$, was determined by the weighted mean $F_{\mathrm{ST}}$ using the infinite-island approximation: $F_{\mathrm{ST}}=1 /\left(1+4 N_{\mathrm{e}} m\right)$ (Wright 1943; Cockerham and Weir 1993). Each coalescent simulation was used to generate a total of 50,000 paired values of $F_{\mathrm{ST}}$ and $H$. The paired values were rank-ordered by $H$ and grouped into overlapping bins of 4,000 points centered on every 2,000th point. For each bin of $F_{\mathrm{ST}}$ values, the first four moments of the distribution were used to generate a Johnson curve (Hill et al. 1976). This curve was then used to compute the $0.975,0.500$, and 0.025 quantiles of the distribution of $F_{\mathrm{ST}}$ as a function of $H$ (Beaumont and Nichols 1996). (Quantiles were computed using a program provided by M. A. Beaumont, University of Reading, U.K.)

To assess how different parameterizations of the coalescent model affect the form of the expected neutral distribution, we performed additional simulations for each dataset that incorporated the following modifications: (1) the number of subpopulations was reduced by half (from 100 to 50), (2) mutational dynamics conformed to the stepwise mutation model rather than the infinite alleles model, and (3) simulated loci were divided into two equal-sized groups of 25,000 that were characterized by a tenfold difference in the scaled mutation rate (either $N_{\mathrm{e}} \mu=0.1$ and $N_{\mathrm{e}} \mu=1.0$, or $N_{\mathrm{e}} \mu=1.0$ and $N_{\mathrm{e}} \mu=10.0$ in each simulation).

To assess the statistical significance of departures from the neutral expectation, two-tailed probabilities were expressed as the proportion of 50,000 simulated values that 
were more extreme than the observed values. For each dataset, the a-level was Bonferroni-adjusted for multiple comparisons $(0.05 /$ number of loci).

\section{Results}

Analysis of allozyme data from a survey of $P$. californicus (Smith 1979) revealed a pronounced degree of genetic subdivision across the species range (weighted mean $F_{\mathrm{ST}}=0.367$, $n=17$ loci). The expected distribution of $F_{\mathrm{ST}}$ as a function of $H$ gave a poor fit to the data, as values for 13 of 17 loci fell below the median quantile of the simulation-based distribution. The poor fit was attributable to two loci, peptidase-1 (Pept-1) and sorbitol dehydrogenase $(S d h)$, which were characterized by exceptionally high levels of differentiation relative to the remaining 15 loci. Probability values for both loci remained highly significant after correcting for multiple comparisons $(P<0.00294)$. The weighted mean $F_{\mathrm{ST}}$ was reduced to 0.234 after removing these two outlying loci, and we used this new average as the expected value for a second set of simulations. $F_{\mathrm{ST}}$ values for Pept-1 and Sdh (0.884 and 0.702 , respectively) still fell far outside the main body of the recalculated distribution, whereas nearly all remaining loci fell within the 0.025 and 0.975 quantiles (Figure 1A).

Analysis of data from a survey of P. furvus (Harris and Rogers 1999) revealed no significant departures from the expected neutral distribution after correcting for multiple comparisons (Figure 1B). However, in each of the remaining datasets, weighted mean $F_{\mathrm{ST}}$ values were substantially reduced by the removal of outlying loci (i.e., those that remained statistically significant after correcting for multiple comparisons; Table 2). For example, in P. gossypinus (Boone 1995; Boone et al. 1999), the weighted mean $F_{\mathrm{ST}}$ was reduced from 0.178 to 0.089 after removing albumin $(A l b)$ and general plasma protein-1 $(P g-1)$. Similarly, in P. maniculatus (Marquardt 1976), the weighted mean $F_{\mathrm{ST}}$ was reduced from 0.050 to 0.019 after removing $A l b$. When the new averages were used as expected values for the simulations, $F_{\mathrm{ST}}$ values for the putatively selected loci still fell far outside the main body of the recalculated distribution, whereas nearly all remaining loci fell within the 0.025 and 0.975 quantiles (Figure 1C, D).

$A l b$ also emerged as one of the most strongly differentiated loci in allozyme surveys of P. leucopus (Robbins et al. 1985; Boone 1995) and P. polionotus (Selander et al. 1971; Figure 1E-G). Considering only those loci that remained statistically significant after correcting for multiple comparisons (Table 2), there were nine loci that exhibited higherthan-expected levels of differentiation (a pattern consistent with spatially varying selection) and only two loci that exhibited lower-than-expected levels of differentiation (a pattern consistent with spatially uniform selection, such as simple overdominant selection). Since the data used in this analysis consisted of counts of alleles rather than counts of diploid genotypes, it was not possible to test for deviations from Hardy-Weinberg equilibrium within sampling localities. Such information could potentially provide additional insight into the mode of selection responsible for shaping the observed patterns of variation. However, none of the original publications reported significant within-sample departures from Hardy-Weinberg genotypic proportions at any of the candidate genes listed in Table 2 (Selander et al. 1971; Marquardt 1976; Smith 1979; Boone 1995, 1999).

Results of the sensitivity analysis indicated that the form of the expected neutral distribution is robust to different parameterizations of the coalescent model, because the same loci were identified as outliers in all of the additional simulations. The mean $F_{\mathrm{ST}}$ of the simulated data tended to fall slightly below the observed weighted mean when the number of demes was reduced by half (from 100 to 50), but the effect was not sufficient to alter any conclusions of the original analyses. Simulations based on the stepwise mutation model and infinite alleles model generated very similar distributions of $F_{\mathrm{ST}}$ as a function of $H$. The only exception was that, when the number of demes was reduced to 50 , the stepwise mutation model predicted a sharper decline in $F_{\mathrm{ST}}$ at $H$ values exceeding approximately 0.8 , consistent with the results of Flint et al. (1999). The sensitivity analysis also indicated that the relationship between $F_{\mathrm{ST}}$ and $H$ is not strongly affected by variation in mutation rate under either mutational model. Higher values of $N_{\mathrm{e}} \mu$ generally predicted a slightly narrower distribution of $F_{\mathrm{ST}}$ at $H$ values lower than approximately 0.2 , consistent with the results of Beaumont and Nichols (1996).

\section{Discussion}

Results of this analysis of allozyme variation in Peromyscus indicate that in a sample of 10-37 protein-coding genes, it is common to find one to three loci that exhibit highly significant departures from the neutral expectation. In many cases, these loci were characterized by $F_{\mathrm{ST}}$ values that fell far outside the main body of the expected neutral distribution. However, after correcting for the effect of extreme outliers, observed $F_{\mathrm{ST}}$ values generally exhibited a remarkably close fit to the simulation-based distributions. This indicates that the majority of loci are simply tracking stochastic demographic processes. Results of the sensitivity analysis indicated that the identification of selected loci was robust to different parameterizations of the coalescent model. Moreover, simulation results of Beaumont and Nichols (1996) indicate that the expected null distribution is robust to a range of different models of equilibrium and nonequilibrium population structure. For example, distributions generated by a nonequilibrium colonization model were indistinguishable from those generated by the island model at $F_{\mathrm{ST}}$ values less than 0.500 (Beaumont and Nichols 1996).

Variation among allozyme genotypes in fitness-related measures of physiological performance has been documented in natural populations of a number of invertebrates and poikilothermic vertebrates (Koehn et al. 1983; Powers et al. 1991; Watt 1994; Mitton 1997; Eanes 1999; Watt and Dean 2000; Schmidt and Rand 2001; Rank and Dahlhoff 2002). However, in homeothermic vertebrates, there is less evidence to suggest that allozyme variation plays a significant role in physiological adaptation (for exceptions, see Leigh Brown 1977; Chappell and Snyder 1984). Results of this analysis suggest that, at least in Peromyscus, diversifying selection on protein polymorphism may not be uncommon.

Two other interesting results emerged from this analysis of Peromyscus allozyme data: (1) departures from neutrality were mostly attributable to spatially varying selection (as 

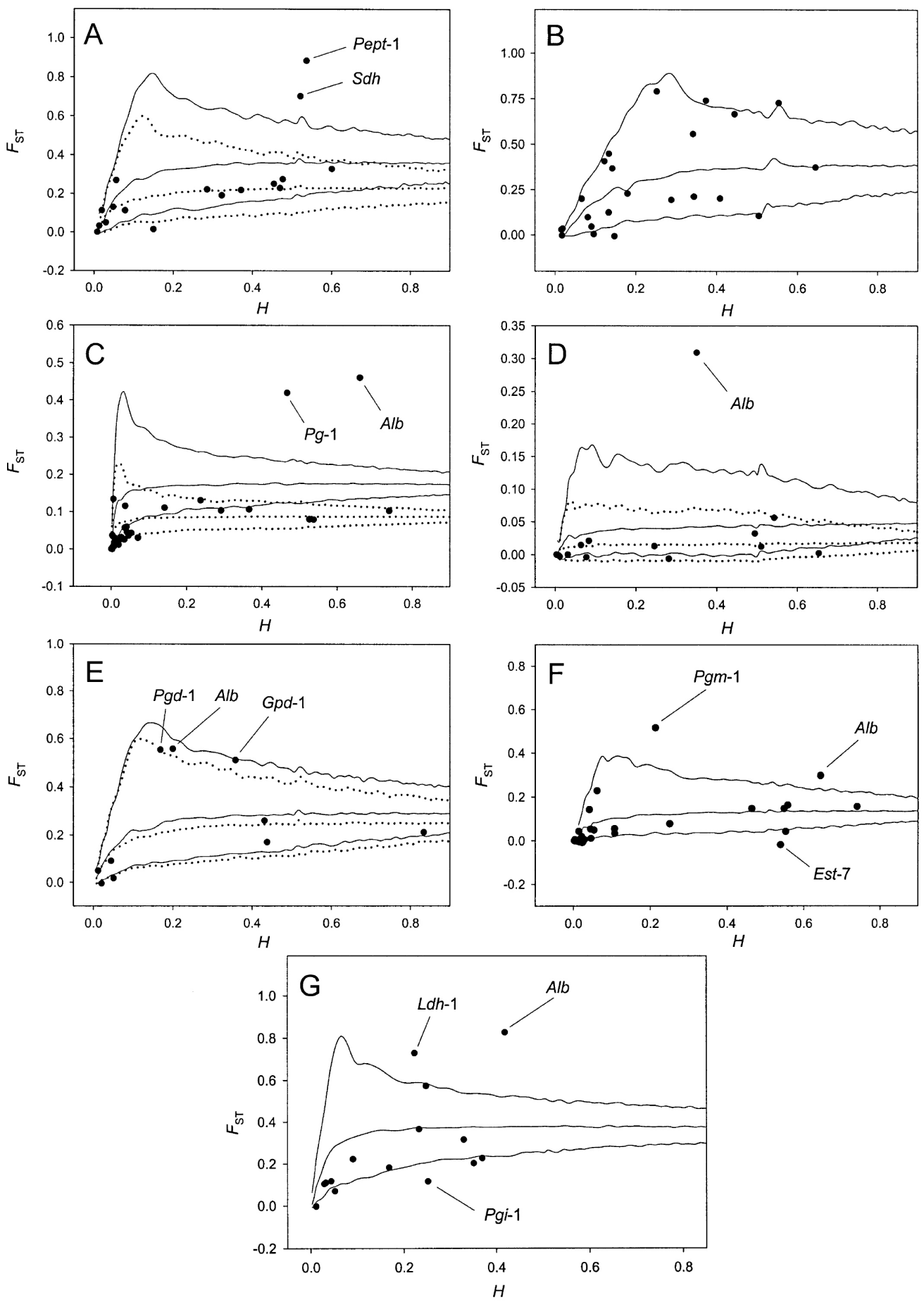

Figure 1. Estimated $F_{\mathrm{ST}}$ values from allozyme loci plotted as a function of heterozygosity in natural populations of Peromyscus. Lines denote the 0.975, 0.500, and 0.025 quantiles of the simulation-based distribution. Dotted lines denote quantiles of the simulation-based distribution that were recalculated after removing outlying loci.

(A) Results from a survey of 17 loci in Peromyscus californicus (Smith 1979; expected $F_{\mathrm{ST}}=0.234$ ). Pept-1 and Sdh exhibited statistically significant departures from the neutral expectation at an adjusted a-level of 0.00294 .

(B) Results from a survey of 24 loci in $P$. furvus (Harris and Rogers 1999; expected $F_{\mathrm{ST}}=0.391$ ).

(C) Results from a survey of 37 loci in P. gossypinus (Boone 1995; Boone et al. 1999; expected $F_{\mathrm{ST}}=0.0 .089$ ). Alb and Pg-1 exhibited statistically significant departures from the neutral expectation at an adjusted a-level of 0.00135 .

(D) Results from a survey of 15 loci in P. maniculatus (Marquardt 1976; expected $F_{\mathrm{ST}}=0.019$ ). Alb exhibited a statistically significant departure from the neutral expectation at an adjusted a-level of 0.00333 .

(E) Results from a survey of 10 loci in P. leucopus (Robbins et al. 1985; expected $F_{\mathrm{ST}}=0.294$ ). Alb, Gpd-1, and Pgd-1 exhibited only marginally significant departures from the neutral expectation at an adjusted a-level of 0.00500 .

(F) Results from a survey of 33 loci in P. leucopus (Boone 1995; expected $F_{\mathrm{ST}}=0.115$ ). Alb, Est-7, and Pgm-1 exhibited statistically significant departures from the neutral expectation at an adjusted a-level of 0.00152 .

(G) Results from a survey of 15 loci in P. polionotus (Selander et al. 1971; expected $F_{\mathrm{ST}}=0.283$ ). Alb, Ldh-1, and Pgi-1 exhibited statistically significant departures from the neutral expectation at an adjusted a-level of 0.00333 . 


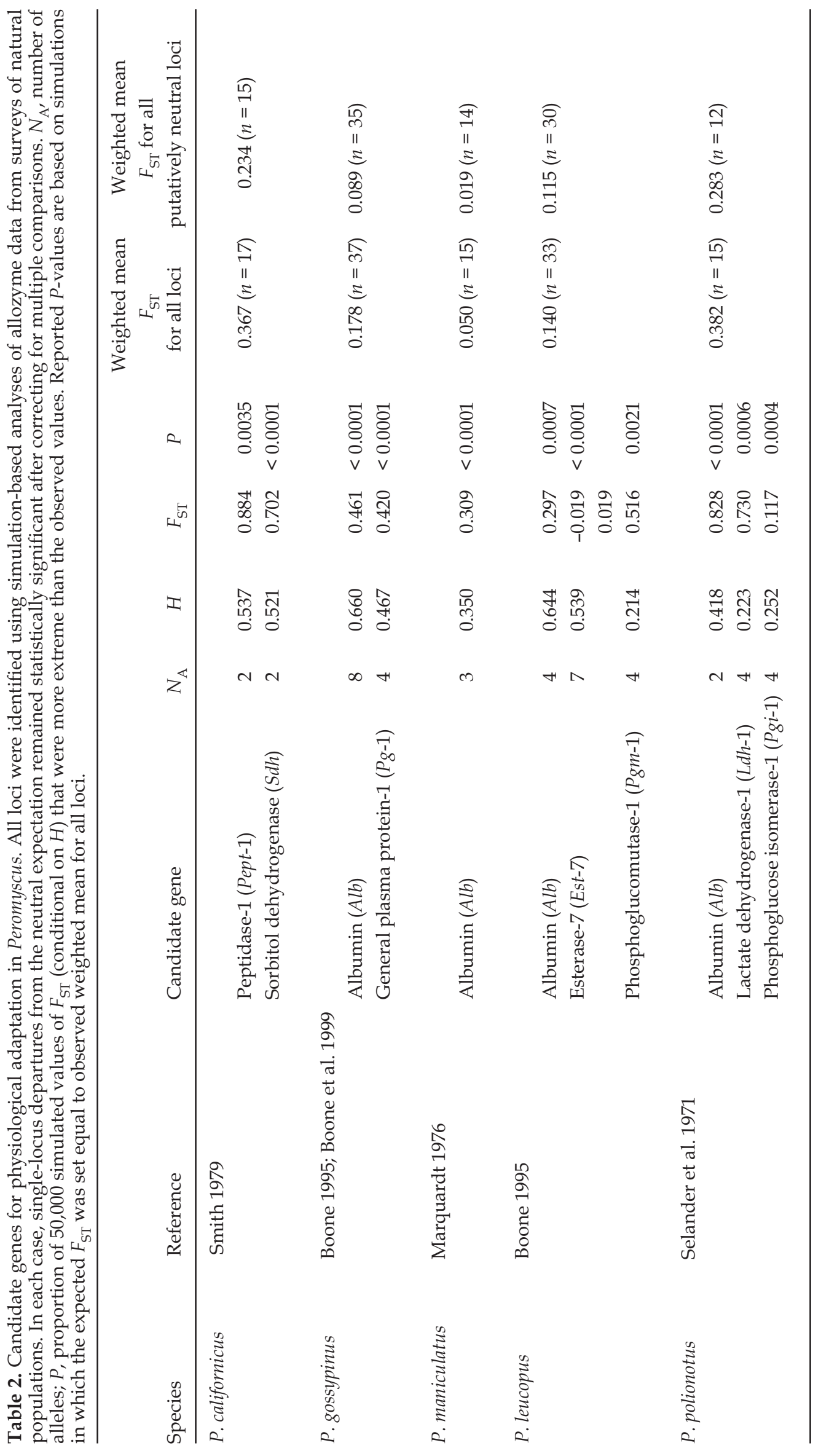


opposed to other forms of balancing selection); and (2) the same locus $(A l b)$ was consistently implicated as a candidate gene for local adaptation. We will discuss each of these observations in turn.

\section{Prevalence of Spatially Varying Selection}

Balanced polymorphisms can be maintained by several different forms of selection. One mechanism is simple overdominance of fitness (single-locus heterosis), which can potentially maintain the same alleles at intermediate frequencies across the entire range of a species. This mode of selection appears to be responsible for the rangewide homogeneity of allele frequencies at the Aat-1 gene (aspartate aminotransferase- $1=$ Got-1) in $P$. maniculatus (Avise et al. 1979; Aquadro and Avise 1982). The two most common Aat-1 electromorphs exhibit remarkably uniform frequencies across North America, in contrast to the pronounced degree of population subdivision revealed by multiple, unlinked allozymes and mtDNA sequence variation (Avise et al. 1979; Aquadro and Avise 1982; Lansman et al. 1983). Electrophoretic variants of Aat-1 segregate as codominant alleles at a single autosomal locus and there is no evidence of mutational heterogeneity within the two most common mobility classes (Aquadro and Avise 1982). If other protein polymorphisms in Peromyscus were maintained by this same mode of balancing selection (i.e., where the fitness rankings of different genotypes are the same in different geographic regions), we would expect most single-locus departures from neutrality to involve an unusually low degree of spatial heterogeneity in allele frequencies. Such loci would be characterized by $F_{\mathrm{ST}}$ values that fall well below the simulation-based expectations. In fact, we observed the opposite pattern: most loci that exhibited a significant departure from neutrality were characterized by higher-than-expected values of $F_{\mathrm{ST}}$. This pattern suggests that diversity-enhancing selection more commonly arises from marginal overdominance of fitness (in which specieswide heterozygosity is maintained at a stable equilibrium even though the heterozygote is not superior to the homozygotes in any particular environment) rather than simple overdominance (in which heterozygous genotypes are unconditionally superior across the full range of different environments). However, we cannot rule out the possibility that this model has relatively more power to detect the effects of diversifying selection.

\section{Evidence for Spatially Varying Selection on Alb Polymorphism}

In mammals, albumin is a soluble, monomeric protein that is highly concentrated in the blood serum. It primarily acts as a transport protein for steroids, fatty acids, and thyroid hormones. It also plays an important role in the regulation of extracellular fluid volume. In Peromyscus, experimental data have confirmed that electrophoretic variants of Alb segregate as codominant alleles at a single autosomal locus (Welser et al. 1965; Brown and Welser 1968; Jensen and Rasmussen 1971; Baccus et al. 1980; Dawson 1982; Dawson et al. 1983). This locus consistently emerged as one of the most highly differentiated loci in the Peromyscus survey data, and after correcting for multiple comparisons, it was characterized by statistically significant departures from neutrality in four species ( $P$. gossypinus, $P$. leucopus, $P$. maniculatus, and $P$. polionotus; Table 2). Inferences about selection on $\mathrm{Alb}$ polymorphism are bolstered by independent evidence from a selection component analysis of motheroffspring genotypes in P. maniculatus (Baccus et al. 1980). It thus seems clear that $A l b$ (or a closely linked locus) is subject to diversifying selection in multiple species of Peromyscus. Parallel patterns of selection at the same loci have also been documented in different species of field crickets (Harrison 1977; Katz and Harrison 1997) and in geographically isolated populations of Drosophila melanogaster (Oakeshott et al. 1982, 1983, 1984; Bubliy et al. 1999).

It is not clear what environmental factors may be mediating selection on $A l b$ polymorphism in natural populations of Peromyscus. Functional studies will be required to determine the mechanistic basis of fitness differences among alternative genotypes. Such studies will also be required to illuminate the adaptive significance of the other putatively selected loci listed in Table 2.

It is possible that the apparent evidence for selection on $A l b$ and other allozyme loci is actually attributable to the indirect effects of selection at closely linked loci. For example, an unusually high level of differentiation at a particular locus could be attributable to hitchhiking with a locally adaptive mutation that has undergone a geographically restricted selective sweep. Likewise, an unusually low level of differentiation could be attributable to linkage with a balanced polymorphism maintained by overdominant selection (associative overdominance). It remains to be seen whether the candidate genes for physiological adaptation in Peromyscus are in fact subject to the direct effects of selection, or whether observed patterns of variation often reflect selection at linked loci. It is also possible that the amino acid polymorphism(s) that underlie mobility differences among electromorphs may be acting as markers for larger segregating units. For example, Adh polymorphism in Drosophila melanogaster and $L d h-b$ polymorphism in Fundulus heteroclitus both appear to fit a multi-site model of epistatic selection (Laurie et al. 1991; Berry and Kreitman 1993; Laurie and Stam 1994; Schulte et al. 1997). Analysis of DNA sequence variation underlying other clinally varying allozymes may ultimately shed more light on the role of epistatic selection in maintaining balanced polymorphism at linked loci.

\section{Interlocus Contrasts and Estimation of Neutral Parameters}

Results of this analysis of Peromyscus allozyme data indicate that the effects of selection can severely bias the estimation of neutral parameters such as $N_{\mathrm{e}} m$. As an illustrative example, consider the reanalysis of Marquardt's (1976) allozyme data for P. maniculatus in Colorado. Using Wright's (1943) equilibrium island-model approximation, the weighted mean $F_{\mathrm{ST}}$ for all 17 loci (0.050) translates into an estimate of $N_{\mathrm{e}} m \approx 4.75$ migrants/generation. After removing the outlying value for Alb (a locus that clearly violates the assumption of neutrality; Figure 1D), the revised $F_{\mathrm{ST}}$ for the remaining 14 loci (0.019) translates into an estimate of $N_{\mathrm{e}} m \approx 12.91$ migrants/generation. Thus, failure to account for the effects of selection at a single locus results in a $63 \%$ reduction in the inferred level of gene flow. Since interlocus contrasts can be used to identify specific loci that 
violate the assumption of neutrality, this type of analysis can play an extremely valuable role in marker-based studies of population structure and gene flow.

\section{Acknowledgments}

We thank M. Beaumont and W. Maddison for helpful discussion and advice regarding the simulation analyses. We also thank M. Beaumont, J. Good, J. Hey, and B. Payseur for helpful comments on an earlier draft of the manuscript. JFS received support from a National Institutes of Health Postdoctoral Fellowship (F32 HL68487-01) and a Fellowship in Computational Molecular Biology from the Alfred P. Sloan Foundation and U. S. Department of Energy.

\section{References}

Aquadro C. F., and J. C. Avise. 1982. An assessment of "hidden" heterogeneity within electromorphs at three enzyme loci in deer mice. Genetics. 102:269-284.

Avise J. C., M. H. Smith, and R. K. Selander. 1979. Biochemical polymorphism and systematics in the genus Peromyscus. VII. Geographic differentiation in members of the truei and maniculatus species groups. J. Mammal. 60:177-192.

Baccus R., J. Joule, and W. J. Kimberling. 1980. Linkage and selection analysis of biochemical variants in Peromyscus maniculatus. J. Mammal. 61:423-435.

Baker R. J., L. W. Robbins, F. B. Stangl Jr., and E. C. Birney. 1983. Chromosomal evidence for a major subdivision in Peromyscus leucopus. J. Mammal. 64:356-359.

Beaumont M. A., and R. A. Nichols. 1996. Evaluating loci for use in the genetic analysis of population structure. Proc. R. Soc. Lond. B. 263:1619-1626.

Berry A., and M. Kreitman. 1993. Molecular analysis of an allozyme cline: alcohol dehydrogenase in Drosophila melanogaster on the east coast of North America. Genetics. 134:869-893.

Blair W. F. 1950. Ecological factors in speciation of Peromyscus. Evolution. 4:253-275.

Boone J. L. 1995. Patterns of temporal and geographic variation in the genetics and morphology of cotton mice (Peromyscus gossypinus). Ph.D. diss. University of Georgia, Athens, GA.

Boone J. L., M. H. Smith, and J. Laerm. 1999. Allozyme variation in the cotton mouse (Peromyscus gossypinus). J. Mammal. 80:833-844.

Bowcock A. M., J. R. Kidd, J. L. Mountain, J. M. Herbert, L. Carotenuto, K. K. Kidd, and L. L. Cavalli-Sforza. 1991. Drift, admixture, and selection in human evolution: a study with DNA polymorphisms. Proc. Natl. Acad. Sci. USA. 88:839-843.

Brown J. H., and C. F. Welser. 1968. Serum albumin polymorphisms in natural and laboratory populations of Peromyscus. J. Mammal. 49:420-426.

Bubliy O. A., B. A. Kalabushkin, and A. G. Imasheva. 1999. Geographic variation of six allozyme loci in Drosophila melanogaster: an analysis of data from different continents. Hereditas. 130:25-32.
Cavalli-Sforza L. L. 1966. Population structure and human evolution. Proc. R. Soc. Lond. B. 164:362-379.

Chappell M. A., and L. R. G. Snyder. 1984. Biochemical and physiological correlates of a-chain hemoglobin polymorphisms. Proc. Natl. Acad. Sci. USA. 81:5484-5488.

Cockerham C. C., and B. S. Weir. 1993. Estimation of gene flow from F-statistics. Evolution. 47:855-863.

Dawson W. D. 1982. Protein polymorphisms in American deer mice (Peromyscus) and genetic linkage homology. Acta Theriol. 27:213-230.

Dawson W. D., L. L. Huang, M. R. Felder, and J. B. Shaffer. 1983. Linkage relationships among eleven biochemical loci in Peromyscus. Biochem. Genet. 21:1101-1114.

Eanes W. F. 1987. Allozymes and fitness: evolution of a problem. Trends Ecol. Evol. 2:44-48.

Eanes W. F. 1999. Analysis of selection on enzyme polymorphisms. Annu. Rev. Ecol. Syst. 30:301-326.

Flint J., J. Bond, D. C. Rees, A. J. Boyce, J. M. Roberts-Thomson, L. Excoffier, J. B. Clegg, M. A. Beaumont, R. A. Nichols, and R. M. Harding. 1999. Minisatellite mutational processes reduce $F_{\mathrm{ST}}$ estimates. Hum. Genet. 105:567-576.

Gillespie J. H. 1991. The causes of molecular evolution. Oxford Univ. Press, New York.

Harris D., and D. S. Rogers. 1999. Species limits and phylogenetic relationships among populations of Peromyscus furvus. J. Mammal. 80:530-544.

Harris H. 1966. Enzyme polymorphisms in man. Proc. R. Soc. Lond. B. 164:298-310.

Harrison R. G. 1977. Parallel variation at an enzyme locus in sibling species of field crickets. Nature. 266:168-170.

Hill I. D., R. Hill, and R. L. Holder. 1976. Fitting Johnson curves by moments. Appl. Stat. 25:180-189.

Hubby J. L., and R. C. Lewontin. 1966. A molecular approach to the study of genic heterozygosity in natural populations. I. The number of alleles at different loci in Drosophila pseudoobscura. Genetics. 54:577-594.

Jensen J. N., and D. I. Rasmussen. 1971. Serum albumins in natural populations of Peromyscus. J. Mammal. 52:508-514.

Katz L. A., and R. G. Harrison. 1997. Balancing selection on electrophoretic variation of phosphoglucose isomerase in two species of field crickets: Gryllus veletis and G. pennsylvanicus. Genetics. 147:609-621.

Kimura M., and T. Ohta. 1971. Theoretical aspects of population genetics. Princeton Univ. Press, Princeton, NJ.

Koehn R. K., A. J. Zera, and J. G. Hall. 1983. Enzyme polymorphism and natural selection. Pp. 115-136 in M. Nei and R. K. Koehn, eds. Evolution of genes and proteins. Sinauer, Sunderland, MA.

Lansman R. A., J. C. Avise, C. F. Aquadro, J. F. Shapira, and S. W. Daniel. 1983. Extensive genetic variation in mitochondrial DNA's among geographic populations of the deer mouse, Peromyscus maniculatus Evolution. 37:1-16.

Laurie C. C., and L. F. Stam. 1994. The effect of an intronic polymorphism on alcohol dehydrogenase expression in Drosophila melanogaster Adh. Proc. Natl. Acad. Sci. USA. 85:5161-5165.

Laurie C. C., J. T. Bridgham, and M. Choudhary. 1991. Associations between DNA sequence variation and variation in 
expression of the Adh gene in natural populations of Drosophila melanogaster. Genetics. 129:489-499.

Leigh Brown A. J. 1977. Physiological correlates of an enzyme polymorphism. Nature. 269:803-804.

Lewontin R. C. 1974. The genetic basis of evolutionary change. Columbia Univ. Press, New York.

Lewontin R. C., and J. L. Hubby. 1966. A molecular approach to the study of genic heterozygosity in natural populations. II. Amount of variation and degree of heterozygosity in natural populations of Drosophila pseudoobscura. Genetics. 54:595-609.

Lewontin R. C., and J. Krakauer. 1973. Distribution of gene frequency as a test of the theory of the selective neutrality of polymorphisms. Genetics. 74:175-195.

Marquardt R. B. 1976. Linkage and selection component analysis of biochemical variants in the deer mouse (Peromyscus maniculatus). M.S. thesis, Univ. of Colorado, Denver, CO.

McDonald J. H. 1994. Detecting natural selection by comparing geographic variation in protein and DNA polymorphisms. Pp. 88-100 in B. Golding, ed. Non-neutral evolution. Chapman and Hall, New York.

Mitton J. B. 1997. Selection in natural populations. Oxford Univ. Press, New York.

Nei M., and T. Maruyama. 1975. Lewontin-Krakauer test for neutral genes. Genetics. 80:395.

Oakeshott J. G., J. B. Gibson, P. R. Anderson, W. R. Knibb, D. G. Anderson, and G. K. Chambers. 1982. Alcohol dehydrogenase and glycerol-3-phosphate dehydrogenase clines in Drosophila melanogaster on different continents. Evolution. 36:86-96.

Oakeshott J. G., G. K. Chambers, J. B. Gibson, W. F. Eanes, and D. A. Willcocks. 1983. Geographic variation in G6PD and PGD allele frequencies in Drosophila melanogaster. Heredity. 50:67-72.

Oakeshott J. G., S. W. McKechnie, and G. K. Chambers. 1984. Population genetics of the metabolically related Adh, Gpdh, and Tpi polymorphisms in Drosophila melanogaster. I. Geographic variation in G6pdh and Tpi allele frequencies in different continents. Genetics. 63:21-29.

Powers D. A., T. Lauerman, D. Crawford, and L. DiMichele. 1991. Genetic mechanisms for adapting to a changing environment. Annu. Rev. Genet. 25:629-659.

Rank N. E., and E. P. Dahlhoff. 2002. Allele frequency shifts in response to climate change and physiological consequences of allozyme variation in a montane insect. Evolution. 56:2278-2289.
Robbins L. W., M. H. Smith, M. C. Wooten, and R. K. Selander. 1985. Biochemical polymorphism and its relationship to chromosomal and morphological variation in Peromyscus leucopus and Peromyscus gossypinus. J. Mammal. 66:498-510.

Robertson A. 1975a. Remarks on the Lewontin-Krakauer test. Genetics. 80:396.

Robertson A. 1975b. Gene frequency distributions as a test of selective neutrality. Genetics. 81:775-785.

Schmidt P. S., and D. M. Rand. 2001. Adaptive maintenance of genetic polymorphism in an intertidal barnacle: habitatand life-stage-specific survivorship of Mpi genotypes. Evolution. 55:1336-1344.

Schulte P. M., M. Gomez Chiarri, and D. A. Powers. 1997. Structural and functional differences in the promoter and 5' flanking region of Ldh-B within and between populations of the teleost Fundulus heteroclitus. Genetics. 145:759-769.

Selander R. K., M. H. Smith, S. Y. Yang, W. E. Johnson, and J. B. Gentry. 1971. Biochemical polymorphism and systematics in the genus Peromyscus. I. Variation in the oldfield mouse (Peromyscus polionotus). Studies in Genetics VI, Univ. Texas. Publ. 7103:49-90.

Smith M. F. 1979. Geographic variation in genic and morphological characters in Peromyscus californicus. J. Mammal. 60:705-722.

Vitalis R., K. Dawson, and P. Boursot. 2001. Interpretation of variation across marker loci as evidence of selection. Genetics. 158:1811-1823.

Watt W. B. 1994. Allozymes in evolutionary genetics - self-imposed burden or extraordinary tool? Genetics. 136:11-16.

Watt W. B., and A. M. Dean. 2000. Molecular-functional studies of adaptive genetic variation in prokaryotes and eukaryotes. Annu. Rev. Genet. 34:593-622.

Weir B. S., and C. C. Cockerham. 1984. Estimating F-statistics for the analysis of population structure. Evolution. 38:1358-1370.

Welser C. F., H. J. Winkelman, E. B. Cutler, and E. Barto. 1965. Albumin variations in the white-footed mouse, Peromyscus Genetics. 52:483.

Wright S. 1943. Isolation by distance. Genetics. 16:97-159. 